\section{Hyperimmunoglobulin E syndrome in two siblings}

\author{
Abdulmohti Hawilo, Inès Zaraa, \\ Sondes Trojjet, Hella Zribi, \\ Rym Cheikh Rouhou, Dalenda El Euch, \\ Mourad Mokni, Amel Ben Osman \\ Dermatology Department, La Rabta \\ Hospital, Faculté de Médecine, Université \\ El Manar, Tunis, Tunisia
}

\begin{abstract}
Hyperimmunoglobulin E recurrent infection syndrome (HIES) is characterized by recurrent skin and lung infections, eczema, elevated serum immunoglobulin E (IgE) levels (>2000 $\mathrm{IU} / \mathrm{mL}$ ), various connective tissue, skeletal, and vascular abnormalities. ${ }^{1}$ We describe herein two brothers with HIES and documented the complications and management of such involvement.
\end{abstract}

\section{Case Report}

A 4-year-old boy from a consanguineous marriage presented with a history of persistent eczematous rash since the age of weeks. No family history of asthma or atopic dermatitis was noted. Physical examination revealed a coarse facial appearance and growth retardation for his age (Figure 1A). There were eczematous patches, plaques and cold abscesses on extremities and scalp (Figure 1B). Pruritic dermatitis was noted in a distribution atypical for true atopic dermatitis (Figure 1C). Cultures of the abscess yielded Staphylococcus aureus. His serum IgE level was $3000 \mathrm{IU} / \mathrm{mL}$ (normal 0-29.2 IU/mL); other immunoglobulin levels were normal. A parent's serum IgE level was markedly elevated. Based on the combination of chronic dermatitis resembling atopic dermatitis, relapsing severe bacterial infections of skin and increased IgE levels, the diagnosis of HIES was confirmed. He was successfully treated with topical corticosteroids, oral oxacillin and low-dose fluconazole. During the succeeding 23 years, he was hospitalized more times (exacerbation of dermatitis, skin and mucosal infections, pruritis) and treated by local and systemic corticosteroids $(0.5 \mathrm{mg} / \mathrm{kg}$ prednisone), emollients, antibiotics, and antihistamines.

His brother (2-year-old boy) presented with a history of recurrent dermatitis and furunculosis since 1 month of age. He was often afflicted with recurrent skin infections, pneumonia, and bronchitis. Cultures for skin abscesses
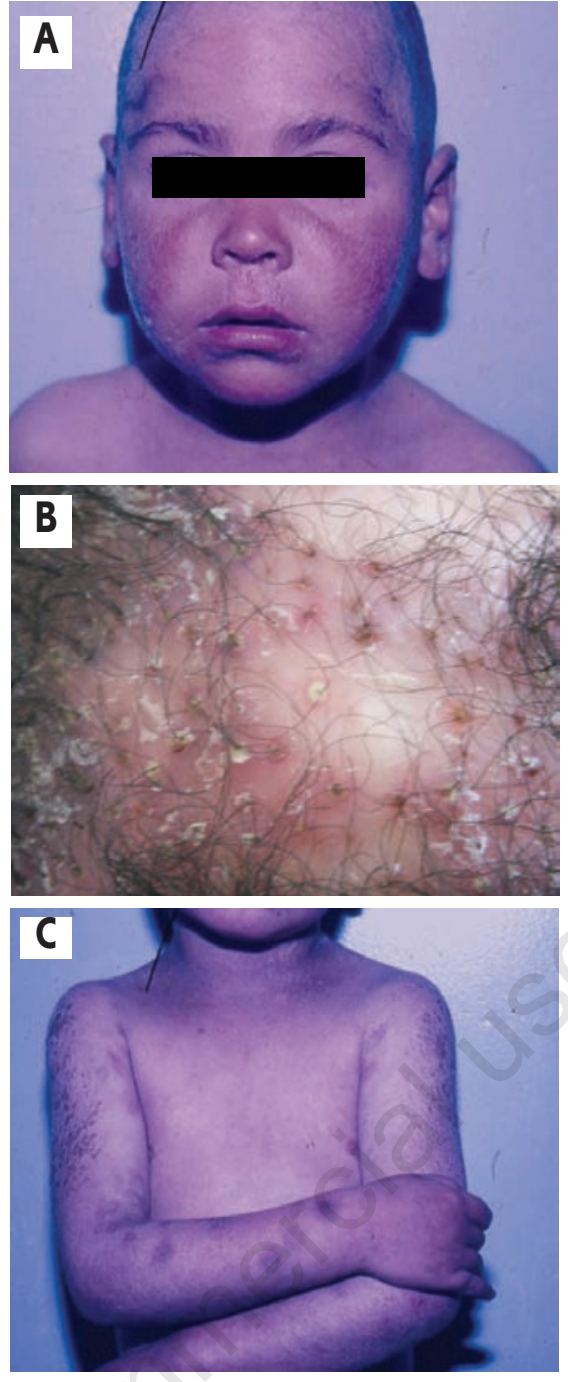

Figure 1. A) A coarse facial appearance with eczematous lesions in a boy with hyperimmunoglobulin E syndrome. B) Folliculitis of the scalp with scaring patches. C) Eczematous skin lesions with excoriations, papules and scaling.

and oral mucosa were positive ( $S$. aureus and C. Albicans). Chemotactic defect in peripheral blood neutrophils was observed. The level of serum IgE was markedly elevated (10000 $\mathrm{IU} / \mathrm{mL}$ ), and anti-S.aureus specific IgE was found. At 5 years of age, he developed typical juvenile dermatomyositis (Figure 2) and treated by oral prednisone $(2 \mathrm{mg} / \mathrm{kg} /$ day $)$. He died at 11 year of age of septic shock infection. Our patients concerned the first two Tunisian cases of HIES, a rare primary immunodeficiency syndrome; less than 250 cases were documented in the literature. ${ }^{1}$ The primary host defense defect is impaired phagocytosis. ${ }^{2,3}$ As observed in patient 2 , serum from HIE patients can inhibit the neutrophilic chemotaxis of healthy patients when added to their serum. Clinical manifestations often start with eczematous or atopic dermatitis-like eruptions within the first days of life as in our patients.
Correspondence: Inés Zaraa, Professor Assistant Address: Dermatology Department, La Rabta Hospital, Jabbari, Bab Saadoun, Tunis, 1007 Tunisia.

Tel. +21.698307425 - Fax: +21.671569449.

E-mail: inesrania@myway.com.

Key words: hyper-IgE syndrome, atopic dermatitis, primary immunodeficiency, infection.

Received for publication: 20 July 2011. Accepted for publication: 12 September 2011.

This work is licensed under a Creative Commons Attribution NonCommercial 3.0 License (CC BYNC 3.0).

() Copyright A. Hawilo et al., 2011

Licensee PAGEPress, Italy

Dermatology Reports 2011; 3:e41

doi:10.4081/dr.2011.e41

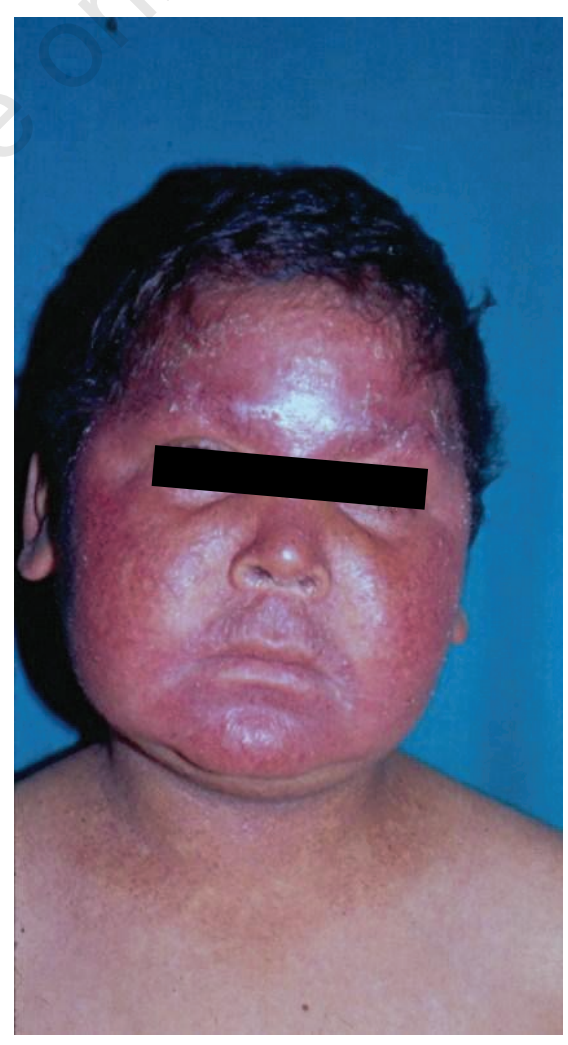

Figure 2. Dermatomyosis in a boy with hyper-immunoglobulin E syndrome.

Recurrent pyogenic pneumonias usually start in early childhood. Other manifestations described since then include: coarse faces as in our first observation, skeletal abnormalities, and vascular abnormalities ${ }^{4}$ (Table 1). The mode of inheritance appears to be autosomal dominant with incomplete penetrance; the gene has been linked to chromosome $4 \mathrm{q}^{5}$ Recently, dominant-negative mutations in the signal transducer and activator of transcrip- 
Table 1. Clinical manifestations and Immunologic features of HIES.

\section{Clinical manifestations}

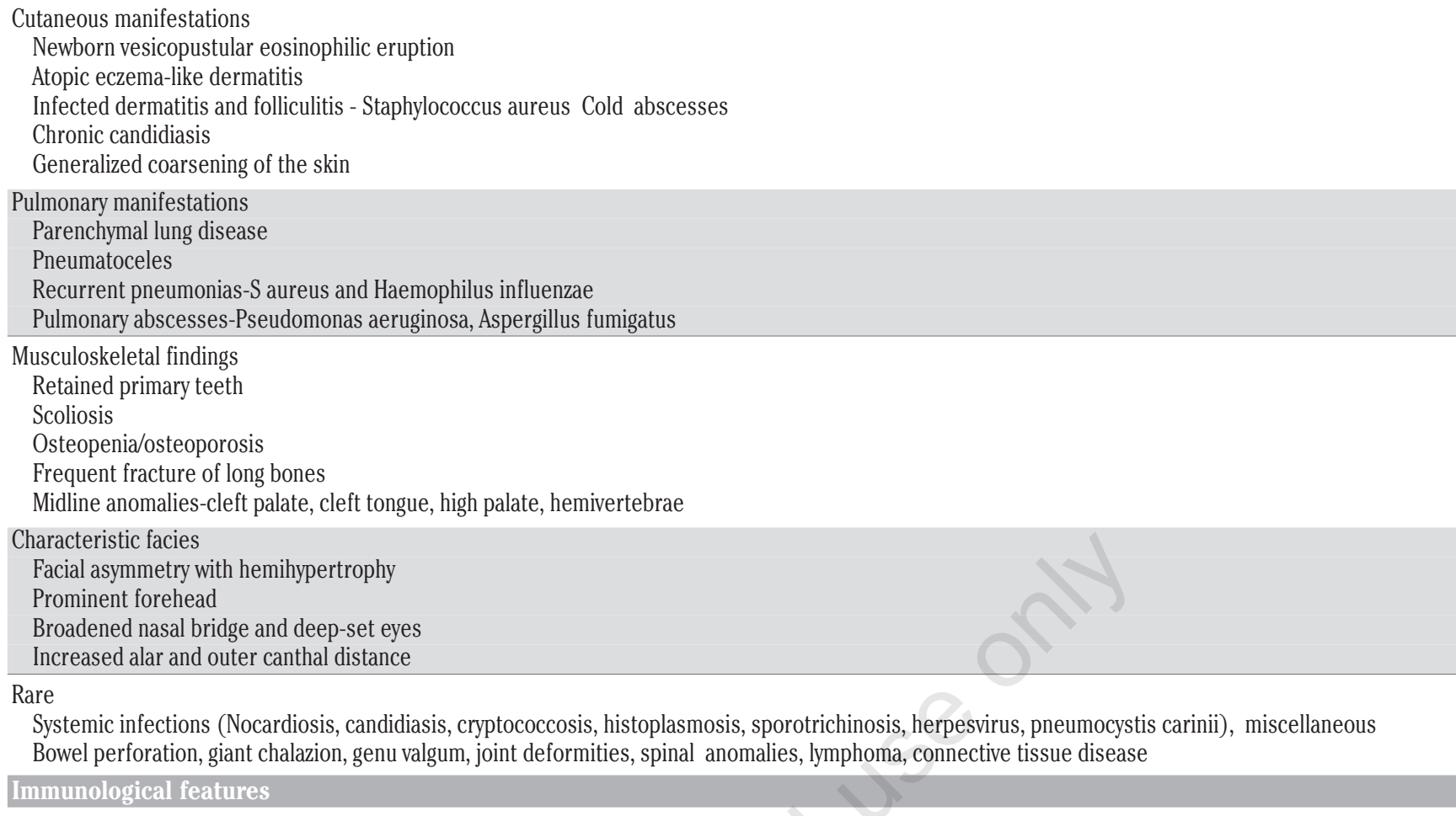

Increased serum IgE, normal IL-4, abnormal neutrophil chemotaxis, decreased C3b receptors on neutrophils, decreased adhesion molecule L-selectin, decreased IFN- $\gamma$ production, poor response to IL-12 stimulation, increased GM-CSF production,decreased TGF- $\beta$

GM-CSF, Granulocyte-monocyte colony-stimulating factor; IFN, Interferon; IL, interleukin; TGF, transforming growth factor.

tion 3 (STAT3) gene were identified. ${ }^{3}$ In this report, the genetic study was not performed. As reported in patient 2 , the association of dermatomyositis with HIES is extremely rare (only 2 reports) and may not have been coincidental. ${ }^{6}$ At present, no established protocol exists for the treatment of HIES. Prevention of infection is the cornerstone of therapy. Bone marrow transplantation has been performed but is likely not fully corrective. ${ }^{7}$ Long-term chemoprophylaxis (oral penicillin's and lowdose fluconazole) in patient 1 improved dramatically the course of the disease.

\section{References}

1. DeWitt CA, Bishop AB, Buescher LS, Stone SP. HyperimmunoglobulinE syndrome: Two cases and a review of the literature. J Am Acad Dermatol 2006; 54:855-65.

2. Minegishi Y. Hyper-IgE syndrome. Curr Opin Immunol 2009;21:487-92.

3. Renner ED, Ryalaarsdam S., AnoverSombke S, et al. Novel signal transducer and activator of transcription 3 (STAT3) mutations, reduced $\mathrm{T}(\mathrm{H}) 17$ cell numbers, and STAT3 phosphorylation in hyper-IgE syndrome. J Allergy Clin Immunol 2008; 122:181-7.

4. Grimbacher B, Holland SM, Gallin JI, et al. Hyper-IgE syndrome with recurrent infections - an autosomal dominant multisystem disorder. N Engl J Med 1999;340:692702 .

5. Grimbacher B, Schaffer AA, Holland SM, et al. Genetic linkage of hyper-IgE syndrome to chromosome 4. Am J Hum Genet 1999; 65:735-44.

7. Saikia B, Aneja H, Jain J, Puliyel JM. Hyperimmunoglobulin E syndrome with juvenile dermatomyositis and calcinosis. Clin Rheumatol 2010 Apr 8. 